\title{
How to Analyse Therapeutic Games: The Player / Game / Therapy Model
}

\author{
Stéphanie Mader ${ }^{\star}$, Stéphane Natkin, and Guillaume Levieux \\ C.E.D.R.I.C. / C.N.A.M., \\ 292 Rue Saint Martin, 75003 Paris, France \\ \{stephanie.mader, stephane.natkin, guillaume.levieux\}@cnam.fr \\ http://cedric.cnam.fr
}

\begin{abstract}
In this paper, we present a new model to analyse therapeutic games. The goal of the model is to describe and analyse the relations between the three aspects of a therapeutic game: the player, the game, and the therapy. The model is intended to game designers. It is a tool to improve the communication between health experts and game designers, and to evaluate the game design coherency of therapeutic games. It also helps to analyse existing games to discover relevant features. The model is built with respect to existing serious game definitions and taxonomies, medical definitions, motivation theory, and game theory. We describe how the model was used to design le village aux oiseaux, a therapeutic game which goal is to train people with attention disabilities. In the last section, we present the results of analysis done with our model and discuss the model limits.
\end{abstract}

Keywords: video games, evaluation, analysis, model, game design, serious games, therapeutic.

\section{Introduction}

"Game design is the process by which a game designer creates a game, to be encountered by a player, from which meaningful play emerges" [29]. Salen and Zimmerman's definition emphasizes that a game can not be dissociated from its player. Neither the player and its relation to the game, nor games fundamentals like gameplay, feedbacks, or goals are stressed in existing definitions of serious game. In this paper, we present a new analysis model for therapeutic games. This model is intended to game designer. They can use the model to evaluate the coherency of their design regarding the relation that bounds the three aspects of a therapeutic game: the therapy, the game, and the player. The goal of the model is also to facilitate the communication between game designers and health experts. Finally, the model can be used to analyse existing therapeutic games to better understand why some of them are efficient while others are not.

\footnotetext{
* Stéphanie Mader gratefully acknowledges the financial support of the Swiss National Science Foundation (SNF).
} 
First, we present how we built the model with respect to existing serious game definitions and taxonomies, medical definitions, motivation theories and game theories. Then, we present our model and explain how it was used during the design of le village aux oiseaux. After that, we present interesting results of analyses made with the model. Finally, we discuss our work and present the next steps of our research.

\section{Background}

\subsection{Serious Games}

According to Sawyer and Smith, the definition of serious game has evolved 30. Old definitions were narrow, they restricted serious games to certain game genres (i.e. simulation-based game) and application fields (e.g. government, education). New definitions are more general, they include all game genres and application fields. Although these definitions are numerous 7 8.11.35, we have identified two main common aspects: a serious game has a purpose beyond entertainment, and the main objective of this combination (serious and game) is to use the attractive shapes of the game to serve the serious purpose. Many other authors also consider that enhancing the user's motivation is the main advantage of serious games over other media $[2,6,10,26$. However, other aspects of serious games are still under discussion. For our research, we use the definition of Guardiola et al.: "A serious game is a rule-based formal system with a variable and quantifiable outcome, where different outcomes are assigned different values, the player exerts effort in order to influence the outcome, the player feels attached to the outcome. But a serious game is combined with a defined real life objective."1 [1]. This definition is based upon the classical game definition of Juul [14. According to Guardiola et al., the main difference between a video game and a serious game is that the designer of a serious game try to have an impact on real life. Thus, the real life consequences, that are optional and negotiable as stated by Juul, become more controlled by the designer in a serious game. If this serious purpose is what differentiate a video game and a serious game, according to existing taxonomies, the serious purpose is also what differentiate a serious game from another one.

Sawyer and Smith propose several taxonomies for serious games [30. Based on market and purpose, their taxonomy split serious games into categories like Games for Education or Game for Health. Then, for each of these categories, Sawyer and Smith propose a specific taxonomy. The taxonomy of Games for Health has two parameters: function (preventative, therapeutic, assessment, educational, informatics) and public (personal, professional practice, research / academia, public health). Djaouti and Alvarez built their own classification

\footnotetext{
1 " un jeu utile est un système dynamique formel dont le comportement, délimité par des règles, produit des conséquences variables et des effets quantifiables. Le joueur doit avoir la sensation que ses actions influencent de façon contrôlée le comportement du jeu. Il doit être émotionnellement attaché aux résultats observés. Mais le jeu utile est associé à un objectif défini de la vie réelle"
} 
upon Sawyer and Smith's taxonomies [1,7]. Djaouti and Alvarez classification of Games for Health is a four-parameter model: gameplay, purpose, function, and public. Function and public parameters are similar to Sawyer and Smith's, except that therapeutic becomes a subitem of the broader function care. The purpose only contains three items: broadcast a message (which can be informative, persuasive, etc.), provide a training, enhance data sharing. Djaouti and Alvarez's work provides information on game features. Their gameplay parameter makes the difference between Ludus and Païda based on the presence or not of explicit goals. Moreover, they work on a general serious game classification which uses their gameplay bricks taxonomy along with game genres and themes keywords [21] 8 .

With the help of these taxonomies, we identified that therapeutic games are serious games which are classified as Games for Health. Their therapeutic function is what makes them different from other Games for Health. Although we did not found a classification specific to therapeutic games, the classification of Alvarez and Djaouti helps to differentiate therapeutic games. Such classification is useful. First, it deepens our understanding of what therapeutic games can achieve. Second, it helps to find similar therapeutic games. Finally, it makes possible to delimit the scope of a therapeutic game during the design steps. Thus, we use this classification to study therapeutic games in the next section.

\subsection{Therapeutic Games}

McGraw-Hill Concise Dictionary of Modern Medicine proposes this definition of a therapy: "A general term for any form of management of a particular condition; treatment intended and expected to alleviate a disease or disorder; any technique of recovery, which may be medical, psychiatric, or psychological." The dictionary also proposes a list of more than two hundreds different therapies. Some of them are very general (e.g. Physical therapy), while others are very specific (e.g. Nicotine replacement therapy). But game designers are seldom medical experts. Thus, it would not be useful for them to classify therapy from a medical standpoint. However, we have to acknowledge the wide scope of possible therapeutic protocol and define a classification useful for game designers.

We have analysed existing therapeutic games and identified that, while some of them are directly therapeutic, others are indirectly therapeutic. For example, the game Brick 'a' break described in Burke et al. is a direct therapeutic games 3, 4]. The game activity is the rehabilitation protocol. Brick 'a' break improves the particular condition of having a loss of motor control in upper limb after a stroke. Equally, playing Virtual Iraq is what directly alleviate or suppress the post traumatic stress of soldiers [27]. Also, le village aux oiseaux aims to reduce the effects due to the disease Alzheimer by training the patient's attentional network.

On contrary, indirect therapies are in themselves not sufficient to improve the particular conditions of a patient. Instead, they improve the patient behaviour,

${ }^{2}$ (c) 2002 by The McGraw-Hill Companies, Inc. 
mood, or observance. For example, RE-Mission is a game for patients having a cancer [15. The game provides knowledge on the disease, its treatment, as well as the means to alleviate the adverse effects. As a result, the patients become more observant with their therapy protocol (i.e. they take more regularly their oral chemotherapy, along with drugs to alleviate the adverse effects). This change in the patient's behaviour make the main therapy more efficient.

Other indirect therapeutic games do not target the patient, but the care giver work, the scientific research or the patient's relatives. For instance, SnowWorld is a virtual reality game that try to overload the attentional network of highlyburned patients. The objective is to alleviate the patient's pain during the bandage change in order to facilitates the care giver's work [12. FoldIt and Phylo are games that help the scientific research to advance on therapeutics by using the computing power of mankind [1] [16. These games aims the gathering of knowledge that could improve the development of therapy. Finally, other therapeutic games target the patient's relatives. For instance, Elude helps the patient's relatives to understand what it means to be depressive [31. These games have an indirect therapeutic effect by engaging the relatives in the recovery process.

We have classified these therapeutic games according with Alvarez and Djaouti's public and purpose parameters.

\begin{tabular}{|c|c|c|}
\hline Name & Public & Purpose \\
\hline Brick 'a' break & Patient / Direct & Provide a training \\
\hline Virtual Iraq & Patient / Direct & Provide a training \\
\hline Le Village aux Oiseaux & Patient / Direct & Provide a training \\
\hline RE-Mission & Patient / Indirect & Broadcast a message \\
\hline FoldIt / Phylo & Everyone / Indirect & Improve data sharing \\
\hline Elude & Patient's relatives / Indirect & Broadcast a message \\
\hline
\end{tabular}

Fig. 1. Classification of some therapeutic games

These different therapeutic uses of games do not require the same design methods. For example, games like RE-Mission or Elude are, from a game design standpoint, similar to Games for Education. They can be designed with existing methods, see 24 or 34 for examples. Also, FoldIt and Phylo are defined as Games for Research, they require a specific design process which is different from, for example, the game design process of le village aux oiseaux or Virtual Iraq. Also, we did not classify SnowWorld. The game has a direct therapeutic effect (i.e. it alleviates temporary the patient's pain) and an indirect therapeutic effect (i.e. it facilitates the care giver's work). But, none of these effects are precisely described with the existing items of Djaouti and Alvarez's purpose parameter. As our objective is to improve the design efficiency, we prefer to treat games like SnowWorld as special case, instead of describing them imprecisely. In fact, the most relevant purpose item is the broadcast of a persuasive message. But the design of persuasive game seems not to be the same as the design required to create a game like SnowWorld. The analyses of these therapeutic games reveal 
that they require very different design process. Thus, we decided to limit the scope of the first version of our model to therapeutic games which provide a training that improve directly the patient condition.

Most of these therapeutic games have been evaluated and have a proven medical efficiency. The fact they are played could indicate that they are good games, but they can also be played only for their therapeutic efficiency (i.e. without pleasure). As we advocate that therapeutic games have to be good games, we study why games are motivating in the next section.

\subsection{Game}

As motivation is the most expected effect of a game in the context of a therapy, the model emphasizes motivational aspects of games. First, we compare play and therapeutic activities regarding motivation theory. Then, we examine game theory with regards to their relation to the player motivation.

Ryan and Deci classify motivation quality as follows: amotivation (i.e. when there is no motivation), extrinsic motivation (i.e. when the activity is done to obtain a separable outcome), and intrinsic motivation (i.e. when the activity is done for the activity itself) [28. They split extrinsic motivation into four subcategories, depending on the level of internalization (i.e. the process of taking in a value or a regulation) and integration (i.e. transformation of the value or regulation to fully integrate the sense of self) one's made of the activity. Also, Ryan and Deci stated that "intrinsic motivation exists in the nexus between a person and a task".

However, Csikszentmihalyi's flow theory explains that some activities can more easily elicit intrinsic motivation [5]. Because games are designed to be pleasurable and interesting activities, games are very powerful to create and foster motivation of their players. Generally, games are played for the sake of playing them. On the contrary, therapeutic activities are designed to maximise their efficiency. Patients engage in therapy for an identified separable outcome: health recovery. Ryan and Deci describe a similar issue in education: "Given that many of the educational activities prescribed in schools are not designed to be intrinsically interesting, a central question concerns how to motivate students to value and self-regulate such activities, and without external pressure, to carry them out on their own." But, according to Ryan and Deci, an activity started for a separable outcome can become a self-determined activity if the person finds the activity sufficiently interesting. We emphasize this and recommend to make a therapeutic game as interesting and fun as possible to smooth out the medical aspect. Doing so should maximise the patient's motivation. As a result, we have now to understand which game features enhance the player motivation.

There exists numerous models of what makes a good video game [6, 18, 19, 23, 25, 33. These models encompass many aspect of video game enjoyment: for instance having an adapted level of challenge, as well as fostering social connectedness or having a high level of fantasy. In this paper, we propose a model for game designers, and moreover, designers of therapeutic games. We will thus 
extract the very common and core features of these models, and especially the features related to the very basis of the game designer's work.

Two features seems to be present in many models and are fundamental to the game designer's work: challenge and variability. Challenge is common to all of the pre-cited models. Malone defines challenges as the fact that the player is uncertain to reach his goals. This is why games have a certain difficulty level: the outcome of the game depends on the player inputs, and not any input may lead the player to his goal. To analyse the challenge level of a game, we may consider different kinds of difficulty that a game may rely on. For instance, Levieux proposes to distinguish between sensitive, logical and motor difficulty to better analyse the challenge level of a task 20. These kinds of difficulty are particularly relevant for therapeutic games. For instance, patients having low motor abilities can have standard logical abilities.

Variability is common to $6,18,23,33$. In his model, Malone presents variability as the curiosity level. It may be sensitive curiosity, that is, the variability of the representation, or the cognitive curiosity, which corresponds to how much information the player can learn when playing a game. Also Vorderer et al explains that a game should propose many choices to the player [33], Ralph Koster that we enjoy learning patterns in games [18, and Denis and Jouvelot that game designers must derail the gameplay to create alternatives for the player [6].

Challenge and variability aspects are tightly related to the design of a therapeutic game. If we want to keep the player motivated, we have to inform him on his progression towards the goal. Another basis is to adapt the challenge level, if we don't want to create anxiety or boredom, or even let the player reach a flow state [5]. And if we want to keep the player motivated in the long run, we must provide enough variability. It is not enough to give the player the same task with an adapted difficulty level, we must also make him learn new patterns, gather new informations, explore the consequences of making different choices.

\subsection{Le village aux oiseaux}

The model presented in this paper has been built during the design of Le village aux oiseaux. Le village au oiseaux is born from a partnership between four companies (Tekneo, Seaside Agency, SpirOps, Neofactory) and two research laboratories (CEDRIC3, INSERM 4). Le village au oiseaux targets patients suffering from the Alzheimer disease. The game stimulates the patient's attention to slow down the cognitive decline due to the disease. Le village au oiseaux is designed to be a full-featured game providing a game world, a story and different gameplay activities. The player takes the role of a photograph that will help the inhabitants of a little town to avoid their town's destruction. The player's mission is to take pictures of rare birds. After the model presentation, we explain how the model has proven useful during the design process of le village aux oiseaux.

\footnotetext{
${ }^{3}$ Center for Research in Computer Science and Telecommunications.

${ }^{4}$ National Institute for Health and Medical Research.
} 


\section{Therapeutic Game Model}

As seen in section 2.2, a therapy targets a particular condition which can be a disease, a disorder or a function to recover. Thus, the context of use and the patient's condition are mandatory parameters to define a therapeutic game. A game is not therapeutic de facto, the therapeutic function emerges from the relation between the player and the game. To illustrate this fact, a good example is Active Music Therapy which uses music as a mediation mean 2. Most of the time, playing music is not a therapeutic activity, but within the specific context of psychotherapy, playing music becomes therapeutic.

Moreover, games are designed to please a specific audience. In consequence, a strong understanding of the therapy, the player, the game, and their underlying relations is necessary to design a therapeutic games. This is the main reason why our analysis model is built around these three aspects: player, game, and therapy. In the following section, we explain the scope of the model and our global goals. Then, we study what is important for each aspects of our analysis model. As these aspects have been thought to be analysed quite independently, the relation between each aspect can be examined to find design inconsistencies. Our analysis model is a list of items to look at for each aspect. To help the game designer to understand which information are relevant, the items proposes examples or questions the analysis has to answer.

\subsection{Model Objectives}

As seen in section 2.2 we decided to limit the scope of our model to therapeutic games which are directly therapeutic, target the patient, and provide a training which can be cognitive or motor. Our model goal is two-fold. First, with our model, we can analyse existing therapeutic games to find their interesting features. Second, the model should facilitate the game designer's work during the design process. At the beginning, the model tells the game designer which information he has to gather, especially medical information. Knowing which information is necessary should improve the communication between game designers and health experts. Then, along the design and development of a therapeutic game, the model helps to examine the coherency of the game design choices. For example, an as-fast-as-you-can scoring system is not a coherent design choice for a game that train fine motor control. In this context, such scoring system could even be harmful for the patient. The model helps to reveal these kind of design inconsistencies. More broadly, the objective of the model is to find the therapeutic constraints. By doing so, the game designer is aware of which game features he has to design very carefully (i.e. which part of the game design is strongly constrained by the therapy). Also, the game designer knows on which game features he can be more creative to provide a pleasurable and interesting game. 


\subsection{Therapy}

As stated before, the therapeutic function emerges from the relation between the player and the therapy. As a result, the most important element of a therapy is what the therapy can improve. Also, the definition of therapy states intended and expected. As a therapy is a medical practice we advocate that this expectation is based on a scientific evaluation of efficiency. However, scientific evaluation is not possible from the very start of the development (i.e. during the conceptual step). During the development, evaluation can be done on prototypes but will generally not be sufficient. When the development is finished, long-term benefits evaluations can start. These facts reveal that being clear-cut on scientific proof of efficiency could be harmful to our model, it would exclude not yet finished therapeutic games. As our model is intended to help the game designer during the design process, we want to propose a smoother approach. So, our model defines in-development game as te be evaluated. However, we want to know if these games are founded on existing scientific evidences (e.g. similar positive effects proven in similar games). Nevertheless, a minimal assessment of efficiency is necessary. Games that have been negatively or not evaluated after their completion are thus determined as not therapeutic by our model.

- Expected short-term therapeutic value: e.g. burning calories, improving attentional network

- Expected long-term therapeutic value: e.g. definitive recovery, life-long supporting therapy

- Scientific proof of efficiency: e.g. long-term effects demonstrated, to be evaluated, discussed

- Scientific references: Do similar useful games features have been scientifically evaluated in other games?

\subsection{Player}

Understanding the player is an important aspect of game design. Thus, numerous studies, either academical or industrial, have been conduced to analyse the player and understand what he likes while playing. But, as stated by Ijsselsteijn et al. 13, while information are numerous about usual targets of video games, there is a lack of information on specific population (e.g. seniors). Although there exists numerous usability guidelines for these population, these guidelines are not informative on what, for example, seniors enjoy while playing or which game genres they prefer. As a result, our player model is limited to demographic information and health condition. For the game designer, the most important is to understand what the target is able to do and learn regarding its health condition. Demographic information serves to know player preferences and interests, as well as knowing which usability guidelines to follow. Also, we advice game designer to create personas 5 of their targeted players. It gives guidance while designing by determining the extreme profiles: a senior who never used a

\footnotetext{
${ }^{5}$ A persona is a description of an imaginary person which represents an entire group.
} 
computer, while another one is fond of new technologies. Using these extreme profiles can make the game designer aware of the wide range of different individuals the game should be able to entertain. It also reveals potential usability issues. Finally, we believe that game designers has to gather more information on the target's interests if these information are not available. For example, the game designer can conduce early playtests with similar games as we explained in another article 22 .

- Age range

- Gender

- Particular conditions: e.g. early states of the Alzheimer disease, motor control loss in upper limb

- Abilities: Regarding their age and particular conditions, which are the knowledge and abilities (i.e. motor control and cognitive functions) of the player? Are they likely to present other particular conditions?

\subsection{Game}

As seen in section 2.3. numerous approach exists on what makes a good game, but we have identified that challenge and variability were very common and important. Thus, we propose to analyse the gameplay of therapeutic games from the challenge (goals, feedbacks, scores, and difficulty) and variability perspectives. Also, information about the input and output systems are relevant elements while designing a game. As some games are recognised to provide positive side-effects, the model asks if the game has features which are known to create positive side-effects (e.g. some games are known to improve the mood or self-esteem of their players).

- Input system: How does the player interact with the game?

- Output system: How does the game convey information?

- Goals: Are there appealing goals? Are they short, mid or long-term goals?

- Feedbacks: Which means are used to communicate with the player? Are they informative on the player performance or progression?

- Score: What does the score mean? (e.g. player performance, player progression, health improvement) Is the score informative on the progression towards mid or long-term therapeutic goals?

- Difficulty: How is the difficulty level chosen? (e.g. adaptive, manually chosen by the player, manually chosen by another person) If adaptive, how does it work? Which parameters of the game are modified by the difficulty level?

- Variability: Does the game propose enough variability? (e.g. the player is always doing the same sequence of actions, the player learns regularly to master new patterns, the player can choose his own path within the game, the player has to create new strategies to progress)

- Usability: What are the minimal abilities and knowledge necessary to play the game? Does the game features tutorials and explanations? 
- Expected positive side-effect: What can the game provide to the player that is not part of the therapy itself?

- Reported serious uses: Have the game or analysed features been used for another serious purpose?

\subsection{Underlying Relations}

The most important part of the analysis process is to validate the design coherency. This coherency is examined by evaluating the relation between each aspects of a therapeutic game: the player, the game, and the therapy. Moreover, each aspect of our model has been thought to be independent. So, the model is useful to evaluate an entertainment game: finding its motivational features, existing serious uses, and possible positive effects.

- Player / Therapy: Does the player have a particular condition that can be improved by the therapy?

- Game / Therapy: What is the context of play? (e.g. at home, with a therapist) What is the place of the game within this context? Which game features are therapeutic? (e.g. the gameplay) Which game features are only motivational means?

- Player / Game: Is the player able to play the game? Is the game enjoyable for the player? Is the game safe for the patient's health?

\subsection{Le village aux oiseaux}

Le village aux oiseaux has been inspired by a scientific study of Green and Bavelier. This study proves that action games, particularly first person shooter (FPS), improve the attention network of their players [9]. Le village aux oiseaux uses these intrinsic effects of FPS to smooth the cognitive decline of patients suffering from the Alzheimer's disease. At the beginning of the project, we had information about the game genre and the player demographic information. However, with the model, we knew that we lacked information about our player's abilities, as well as which features of FPS games are effectively training the attention network. Here, to illustrate how our model can work while designing a therapeutic game, we explain two main design decisions the model helped us to make.

First, the analysis of the player-game relation makes clear from the very start that it would not be possible for seniors to play a standard FPS. These games require a strong ability to move in a virtual 3D space. We assumed that starting a therapeutic game by having to learn a difficult ability would alienate our players. So, we decided that Le village aux oiseaux would be a rail shooter (i.e. the player movement are automated and he can focus on the aim and shot activity), and that the player will aim with a natural controller (i.e. similar to the Nintendo's wiimote). However, we had no data to prove that seniors may enjoy a game were they have to aim and shoot with a natural controller. In consequence, we conduced early playtests with similar games to be sure that seniors will enjoy 
the game we were designing. These playtests and results have been detailed in another article 22.

Second, as the gameplay was the therapeutic part of the game, it was really important to know better what was therapeutic in it. This aspect required to exchange with health experts. To sum up, the main concept that health experts make us understand was that if the player can use his memory (i.e. instead of processing the game environment) the therapeutic gameplay fails at training the attention. But, challenge and scoring system of rail shooters are strongly tied to the memorisation of the game sequences. The long-term player's objective is to perform each sequence perfectly. We changed this aspect of the game to make it therapeutic. This is why le village aux oiseaux features adaptive levels, in which the birds act differently each time. We knew that this change had consequences on the long-term motivation provided by the game. With adaptive levels, the player can't dedicate himself to perform perfectly the game sequences. So, to compensate the loss of these long-term goals and strengthen the patient's motivation, we had to design other features (e.g. storyline, collection of pictures, rare birds to discover).

In conclusion, the model helped us to find design inconsistencies. It also revealed the consequences of each game design change and helped us to design more efficiently.

\section{Discussion}

So far, we used our model to analyse five entertainment games and therapeutic games. From these analyses, we draw some early conclusions on the model usefulness. First, the model can be used to determine if a particular use of a game is therapeutic or not. For instance, Brain Age has some proven effects on the brain (e.g. memory improvement). But Brain Age is not therapeutic, because it does not target any particular medical conditions. Equally, the game Ico has been designed for entertainment. Within the specific context of a psychotherapy, Ico can be used as a mediation tool with children having a particular psychology condition [32]. This specific use of Ico is therapeutic. With our model, we can analyse Ico has an entertainment game, but we can also analyse the specific therapeutic use of ICO. Finally, using the model is efficient to find similarities and differences between an entertainment game and a therapeutic games. For example, Dance Dance Revolution and Dance Town feature the same gameplay. Dance Dance Revolution (DDR) is an entertainment rhythm game where the player has to step on buttons according to arrow patterns displayed on a screen. DDR targets adolescents and young adults. On the contrary, Dance Town targets seniors to improve their coordination and balance. We have found that Dance Town features three main adaptations: more protection around the dance pad, songs and visuals that please the seniors, and easier arrow patterns (both in rythm and complexity). These adaptations aims to make the game playable for seniors, but also pleasurable by featuring, for instance, songs they like. In conclusion, the effect of improving coordination and balance is already present in 
the original DDR, game designers of Dance Town have mainly adapted DDR for the specific target.

However, we have also found weaknesses in our model. First, the model asks the game designer to write full-text description for each items. This is very useful and usable for game designers, but not very efficient to conduce automated metaanalyses. Also, we limited the quantity of items in each aspects trying to keep only essential ones. We have now to conduce more analyses to be sure that these items are sufficient. We think that analysing more games like Virtual Iraq could reveal other essential game features (e.g. environment, story).

The next step of our research will be the development of a tool. This tool has the objective to facilitate the use of our model. Also, it will help to structure data in order to conduce meta-analyses later. After that, we will analyse numerous existing therapeutic games to better understand how they are designed and how the relations between the player, the game, and the therapy work. This analysis model and the building of more knowledge is the first step of our research, our final objective is to provide methods to design therapeutic games more efficiently.

\section{Conclusion}

In this paper, we have presented how we built an analysis model for therapeutic games. The model proposes to analyse separately each aspect of a therapeutic games: the player, the game, and the therapy. The objective of the model is to help a game designer while designing a therapeutic games: gathering information with medical experts, examining the game design coherency by analysing the relations between each aspect, evaluating the game efficiency regarding the two objectives of a therapeutic game (i.e. being therapeutic and motivating), and analysing similar games to find their interesting features and how they work regarding therapeutic activity.

During the design of le village aux oiseaux, the model has proven useful. It helped us to make design decision and predict consequences of game design changes. Also, by conducing some analyses, we have found that our model was able to deepen our knowledge on therapeutic games. Separating the three aspects of therapeutic games (i.e. player, game, and therapy), to then analyse their relations has been powerful to understand how therapeutic games work. Also, we have used our model to make comparison between similar games to better understand the effects of certain games features. However, we need a tool to improve the analysis model usability and to obtain structured data on therapeutic games. The development of this tool is the next step of our research, then we will deepen even more our knowledge on therapeutic games. Finally, our objective is to build design methods alongside this analysis model. 


\section{References}

1. Alvarez, J., Djaouti, D.: Une taxinomie des serious games dédiés au secteur de la santé. Revue de l'Electricité et de l'Electronique, Société de l'Electricité, de l'Electronique et des Technologies de l'Information et de la Communication (SEE) 11, 91-102 (2008)

2. Benveniste, S., Jouvelot, P., Lecourt, E., Michel, R.: Designing wiimprovisation for mediation in group music therapy with children suffering from behavioral disorders. In: Proceedings of the 8th International Conference on Interaction Design and Children, IDC 2009, pp. 18-26. ACM, New York (2009)

3. Burke, J.W., McNeill, M.D.J., Charles, D.K., Morrow, P.J., Crosbie, J.H., McDonough, S.M.: Optimising engagement for stroke rehabilitation using serious games. Vis. Comput. 25, 1085-1099 (2009)

4. Burke, J.W., McNeill, M.D.J., Charles, D.K., Morrow, P.J., Crosbie, J.H., McDonough, S.M.: Augmented reality games for upper-limb stroke rehabilitation. In: Proceedings of the 2010 Second International Conference on Games and Virtual Worlds for Serious Applications, VS-GAMES 2010, pp. 75-78. IEEE Computer Society, Washington, DC (2010)

5. Csikszentmihalyi, M.: Flow: The Psychology of Optimal Experience. Harper Perennial (March 1991)

6. Denis, G., Jouvelot, P.: Motivation-driven educational game design: Applying best practices to music education. In: Proc. Int. Conf. on Advances in Computer Entertainment Technology, pp. 462-465. ACM Press (2005)

7. Djaouti, D.: Serious Game Design Considérations théoriques et techniques sur la création de jeux vidéo á vocation utilitaire. $\mathrm{PhD}$ thesis, Université Toulouse III Paul Sabatier (UT3 Paul Sabatier) (2011)

8. Djaouti, D., Alvarez, J., Ghassempouri, R., Jessel, J.P., Methel, G.: Towards a classification of video games. In: Artificial and Ambient Intelligence convention (Artificial Societies for Ambient Intelligence) (2007)

9. Green, C.S., Bavelier, D.: Action video game modifies visual selective attention. Nature 423(6939), 534-537 (2003)

10. Greitzer, F.L., Kuchar, O.A., Huston, K.: Cognitive science implications for enhancing training effectiveness in a serious gaming context. J. Educ. Resour. Comput. 7 (November 2007)

11. Guardiola, E., Natkin, S., Soriano, D., Loarer, E., Vrignaud, P.: Du jeu utile au jeu sérieux (serious game). Hermés 62, 87-93 (in press, 2012)

12. Hoffman, H.G., Doctor, J.N., Patterson, D.R., Carrougher, G.J., Furness, T.A.: Virtual reality as an adjunctive pain control during burn wound care in adolescent patients. Pain (85), 305-309 (2000)

13. Ijsselsteijn, W., Nap, H.H., de Kort, Y., Poels, K.: Digital game design for elderly users. In: Proceedings of the 2007 Conference on Future Play, Future Play 2007, pp. 17-22. ACM, New York (2007)

14. Juul, J.: In: The Game, the Player, the World: Looking for a Heart of Gameness, vol. 120, pp. 30-45. Utrecht University (2003)

15. Kato, P.M., Cole, S.W., Bradlyn, A.S., Pollock, B.H.: A video game improves behavioral outcomes in adolescents and young adults with cancer: A randomized trial. Pediatrics 122(2), e305-e317 (2008)

16. Kawrykow, A., Roumanis, G., Kam, A., Kwak, D., Leung, C., Wu, C., Zarour, E., Sarmenta, L., Blanchette, M., Waldispühl, J.: Phylo: A citizen science approach for improving multiple sequence alignment. PLoS ONE 7(3), e31362 (2012) 
17. Khatib, F., Cooper, S., Tyka, M., Xu, K., Makedon, I., Popović, Z., Baker, D., Players, F.: Algorithm discovery by protein folding game players. Proceedings of the National Academy of Sciences 108(47), 18949-18953 (2011)

18. Koster, R.: A Theory of Fun for Game Design. Paraglyph Press, Scottsdale (2005)

19. Lazzaro, N.: Why we play games: Four keys to more emotion without story. In: Game Developers Conference (March 2004)

20. Levieux, G.: Mesure de la difficulté dans les jeux vidéo. PhD thesis, Conservatoire National des Arts et Métiers (2011)

21. Ludoscience: Serious game classification, http://serious.gameclassification.com (last access June 08, 2011)

22. Mader, S., Dupire, J., Guardiola, E., Natkin, S.: Conception de jeux thérapeutiques pour seniors: l'exemple du village aux oiseaux. In: Handicap 2012, Paris (June 2012)

23. Malone, T.W.: What makes things fun to learn? heuristics for designing instructional computer games. In: SIGSMALL 1980: Proceedings of the 3rd ACM SIGSMALL Symposium and the first SIGPC Symposium on Small Systems, pp. 162169. ACM, New York (1980)

24. Marne, B., Huynh Kim Bang, B., Labat, J.M.: Articuler motivation et apprentissage grâce aux facettes du jeu sérieux. In: Environnements Informatiques Pour l'Apprentissage Humain, pp. 69-80 (May 2011) ISBN: 978-2-87325-061-4

25. McGonigal, J.: Reality is broken. Penguin Books, New York (2011)

26. Prensky, M.: Digital game-based learning. Comput. Entertain. 1, 21 (2003)

27. Rizzo, A., Difede, J., Rothbaum, B., Reger, G., Spitalnick, J., Cukor, J., Mclay, R., et al.: Development and early evaluation of the virtual iraq/afghanistan exposure therapy system for combat-related ptsd. Annals of the New York Academy of Sciences 1208(1), 114-125 (2010)

28. Ryan, R.M., Deci, E.L.: Intrinsic and extrinsic motivations: Classic definitions and new directions. Contemporary Educational Psychology 25(1), 54-67 (2000)

29. Salen, K., Zimmerman, E.: Rules of Play: Game Design Fundamentals. The MIT Press (October 2003)

30. Sawyer, B., Smith, P.: Serious game taxonomy. In: The Serious Games Summit @ GDC (2008)

31. Singapore MIT GAMBIT Game Lab: Elude, http://gambit.mit.edu/loadgame/elude.php (last access March 29, 2012)

32. Stora, M.: Histoire d'un atelier jeu vidéo: " ico ", un conte de fée interactif pour des enfants en manque d'interactions (2008), http://www.omnsh.org/spip.php?article84 (last access June 08, 2011)

33. Vorderer, P., Hartmann, T., Klimmt, C.: Explaining the enjoyment of playing video games: the role of competition. In: ICEC 2003: Proceedings of the Second International Conference on Entertainment Computing, Pittsburgh, PA, USA, Carnegie Mellon University, pp. 1-9 (2003)

34. Wei, T., Li, Y.: Design of Educational Game: A Literature Review. In: Pan, Z., Cheok, A.D., Müller, W., Zhang, X., Wong, K. (eds.) Transactions on Edutainment IV. LNCS, vol. 6250, pp. 266-276. Springer, Heidelberg (2010)

35. Zyda, M.: From visual simulation to virtual reality to games. Computer $38(9)$, 25-32 (2005) 